\title{
A PRODUCT THEOREM FOR $H$-GROUP FIBRATIONS
}

\author{
F. H. CROOM
}

\begin{abstract}
Let $(E, p, B)$ and $\left(E^{\prime}, p^{\prime}, B\right)$ be $H$-group fibrations over $B$ with basic fibers $F$ and $F^{\prime}$ respectively. If there are base point preserving fiber maps $f: E \rightleftarrows E^{\prime}: g$ such that $f$ is a fiber $H$-homomorphism, then $E \times F^{\prime}$ and $E^{\prime} \times F$ have the same homotopy type.
\end{abstract}

1. Introduction. Let $(E, p, B)$ and $\left(E^{\prime}, p^{\prime}, B\right)$ be fiber structures satisfying the weak covering homotopy property and having basic fibers $F$ and $F^{\prime}$ respectively. If these fibrations have certain $H$-group properties and there are fiber maps $f: E \rightleftarrows E^{\prime}: g$ such that $f$ is an $H$-homomorphism, this paper shows that the product spaces $E \times F^{\prime}$ and $E^{\prime} \times F$ have the same homotopy type. If the restriction $\left.f\right|_{F}: F \rightarrow F^{\prime}$ is a homotopy equivalence, it follows that $f$ is a fiber homotopy equivalence. This analogue of Dold's fiber homotopy equivalence theorem [2, Theorem 6.3] requires no local contractibility property of the base $B$.

Under more restrictive hypotheses it is proved that $f$ is a fiber homotopy equivalence provided that it induces a homotopy equivalence on the spaces of based loops in $E$ and $E^{\prime}$.

\section{2. $H$-group fibrations.}

Definition. A sequence $X_{\rightarrow}^{*} Y_{\rightarrow}^{g} Z$ of topological spaces with base points and continuous maps is exact means that

(1) the composition $g f$ is null-homotopic (i.e., homotopic to the constant map whose only value is the base point of $Z$ ); and

(2) for each space $W$ and continuous map $h: W \rightarrow Y$ such that $g h$ is nullhomotopic, there is a continuous map $h_{*}: W \rightarrow X$ such that $f h_{*} \sim h$ (homotopic).

Definition. A space $X$ with continuous multiplication • is an $H$-group means that

(1) there is a point $x_{0} \in X$ such that the maps defined by multiplication on the left and on the right by $x_{0}$ are homotopic to the identity map id $X$ on $X$;

Presented to the Society, January 21, 1971; received by the editors March 12, 1971. AMS 1970 subject classifications. Primary 55F05; Secondary 55D10, 55D35, 55D45.

Key words and phrases. Weak covering homotopy property, $H$-group, fiber homotopy equivalence, loop space.

(c) American Mathematical Society 1972 
(2) the multiplication on $X$ is homotopy associative; and

(3) there is a continuous map $j: X \rightarrow X$ such that the product maps $j \cdot \mathrm{id}_{X}$ and $\mathrm{id}_{X} \cdot j$ are homotopic to the constant map $c\left(x_{0}\right)$ whose only value is $x_{0}$.

The point $x_{0}$ is called a homotopy unit for $X$ and $j$ is called an inversion. If $X$ and $Y$ are $H$-groups, a continuous map $q: X \rightarrow Y$ is an H-homomorphism provided that the maps $\left(x_{1}, x_{2}\right) \rightarrow q\left(x_{1} \cdot x_{2}\right)$ and $\left(x_{1}, x_{2}\right) \rightarrow q\left(x_{1}\right)$. $q\left(x_{2}\right)$ from $X \times X$ to $Y$ are homotopic.

Note. The functions involved in this paper are not assumed to be base point preserving unless specifically stated and it is not assumed that homotopies preserve base points at each level. All function spaces are assigned the compact open topology.

Definition. Let $p:\left(E, e_{0}\right) \rightarrow\left(B, b_{0}\right)$ be a base point preserving map with the weak covering homotopy property and basic fiber $F=p^{-1}\left(b_{0}\right)$. Then $(E, p, B)$ is an $H$-group fibration provided that $E$ and $F$ are $H$-groups with homotopy unit $e_{0}, B$ is an $H$-group with homotopy unit $b_{0}$, and the inclusion $i: F \rightarrow E$ and the projection $p: E \rightarrow B$ are $H$-homomorphisms. If $(E, p, B)$ and $\left(E^{\prime}, p^{\prime}, B\right)$ are $H$-group fibrations over the same base $B$, a fiber map $f: E \rightarrow E^{\prime}$ is a fiber $H$-homomorphism provided that $f$ is an $H$ homomorphism from $E$ into $E^{\prime}$ and the restriction $f^{\prime}=\left.f\right|_{F}: F \rightarrow F^{\prime}$ is an $H$ homomorphism on the basic fibers.

For the remainder of this paper, $(E, p, B)$ and $\left(E^{\prime}, p^{\prime}, B\right)$ will denote $H$ group fibrations over the same space $B$ with basic fibers $F=p^{-1}\left(b_{0}\right)$ and $F^{\prime}=p^{\prime-1}\left(b_{0}\right)$ respectively where $b_{0}$ is the base point of $B$. In accordance with the definition, each of the $H$-groups $E, F, E^{\prime}, F^{\prime}$, and $B$ has its base point as homotopy unit. For $E$ and $F$ the base point will be denoted by $e_{0}$, and for $E^{\prime}$ and $F^{\prime}$ the base point will be denoted by $e_{0}^{\prime}$.

THEOREM 1. Let $(E, p, B)$ and $\left(E^{\prime}, p^{\prime}, B\right)$ be H-group fibrations over $B$ and $f: E \rightarrow E^{\prime}$ a base point preserving fiber H-homomorphism. If there is a base point preserving fiber map $g: E^{\prime} \rightarrow E$ then $E \times F^{\prime}$ and $E^{\prime} \times F$ have the same homotopy type.

Theorem 1 will be established by applying the following result [1, Theorem 1] to a suitably chosen exact sequence:

THEOREM 2. Suppose that the sequence

$$
0 \longrightarrow A \stackrel{q}{\longrightarrow} C \stackrel{r}{\longrightarrow} B
$$

is exact, $r$ has a right homotopy inverse $\tau: B \rightarrow C, A$ and $C$ are $H$-groups with base points as homotopy units, and $q: A \rightarrow C$ is an H-homomorphism. If the map $s: C \rightarrow B$ defined by

$$
s(x)=r(x \cdot j \tau r(x)), \quad x \in C,
$$


where $j$ is the inversion on $C$, is null-homotopic and the map $m: B \times A \rightarrow B$ defined by

$$
m(b, a)=r(q(a) \cdot \tau(b)), \quad(b, a) \in B \times A,
$$

is homotopic to the projection $\Pi_{1}$ on the first component, then $(C, r, B)$ and $\left(B \times A, \Pi_{1}, B\right)$ have the same homotopy type.

Proof of Theorem 1. The desired sequence is

$$
0 \longrightarrow F \stackrel{\theta}{\longrightarrow} E \times F^{\prime} \stackrel{\varphi}{\longrightarrow} E^{\prime}
$$

where $\theta: F \rightarrow E \times F^{\prime}, \varphi: E \times F^{\prime} \rightarrow E^{\prime}$ are defined by

$$
\begin{gathered}
\theta(x)=\left(i(x), f^{\prime}(x)\right), \quad \varphi\left(e, x^{\prime}\right)=j i^{\prime}\left(x^{\prime}\right) \cdot f(e), \\
x \in F, \quad\left(e, x^{\prime}\right) \in E \times F^{\prime} .
\end{gathered}
$$

Here $i^{\prime}: F^{\prime} \rightarrow E^{\prime}$ is the inclusion, $f^{\prime}: F \rightarrow F^{\prime}$ is the restriction of $f, \cdot$ denotes the $H$-group operation, and $j$ denotes inversion.

It is easily observed that the composition $\varphi \theta$ is null-homotopic. Some preliminary observations will be required before proving that the sequence has the required lifting properties.

Consider the sequence

$$
\Omega E \stackrel{i}{\longrightarrow} \Omega(E, F) \stackrel{m}{\longrightarrow} F \stackrel{i}{\longrightarrow} E \stackrel{p}{\longrightarrow} B
$$

where $\Omega E$ is the space of based loops in $E, \Omega(E, F)$ is the space of paths in $E$ with initial point $e_{0}$ and terminal point in $F, m$ denotes evaluation at the terminal point and $i$ denotes inclusion maps. This sequence and the corresponding sequence

$$
\Omega E^{\prime} \stackrel{i^{\prime}}{\longrightarrow} \Omega\left(E^{\prime}, F^{\prime}\right) \stackrel{m^{\prime}}{\longrightarrow} F^{\prime} \stackrel{i^{\prime}}{\longrightarrow} E^{\prime} \stackrel{p^{\prime}}{\longrightarrow} B
$$

for $\left(E^{\prime}, p^{\prime}, B\right)$ are exact.

Let $P E$ denote the space of paths in $E$ with initial point $e_{0}, \Pi: P E \rightarrow E$ the evaluation at the terminal point and

$$
f_{1}: \Omega(E, F) \rightleftarrows \Omega\left(E^{\prime}, F^{\prime}\right): g_{1}, \quad \bar{f}: P E \rightleftarrows P E^{\prime}: \bar{g}
$$

the maps defined from $f$ and $g$ by composition. Then $(P E, p \Pi, B)$ and $\left(P E^{\prime}, p^{\prime} \Pi^{\prime}, B\right)$ have the weak covering homotopy property and the total spaces $P E$ and $P E^{\prime}$ are contractible. It follows from [2, Theorem 6.1] that $\bar{f}$ is a fiber homotopy equivalence. Examination of the proof of that theorem shows that $\bar{g}$ is a fiber homotopy inverse for $\bar{f}$. In particular, the restrictions $f_{1}: \Omega(E, F) \rightleftarrows \Omega\left(E^{\prime}, F^{\prime}\right): g_{1}$ are mutual homotopy inverses. This is not surprising since $\Omega(E, F)$ and $\Omega\left(E^{\prime}, F^{\prime}\right)$ are both homotopy equivalent to $\Omega B$. 
Suppose now that $Y$ is a space and $h=\left(h_{1}, h_{2}\right): Y \rightarrow E \times F^{\prime}$ is a continuous map such that $p h$ is null-homotopic. Then $i^{\prime} h_{2}$ is homotopic to $f h_{1}$ and

$$
p h_{1}=p^{\prime} f h_{1} \sim p^{\prime} i^{\prime} h_{2} \sim 0 .
$$

Hence $p h_{1}$ is null-homotopic and there is a continuous map $t: Y \rightarrow F$ such that $i t \sim h_{1}$. Then

$$
i^{\prime}\left(j h_{2} \cdot f^{\prime} t\right) \sim j i^{\prime} h_{2} \cdot i^{\prime} f^{\prime} t=j i^{\prime} h_{2} \cdot f i t \sim j i^{\prime} h_{2} \cdot f h_{1} \sim 0
$$

and there is a continuous map $\gamma: Y \rightarrow \Omega\left(E^{\prime}, F^{\prime}\right)$ such that $m^{\prime} \gamma$ is homotopic to $j h_{2} \cdot f^{\prime} t$. The required homotopy lifting $h_{*}$ of $h$ is given by

$$
h_{*}=t \cdot j m g_{1} \gamma: Y \rightarrow F \text {. }
$$

To see this, observe that

$$
\begin{aligned}
\theta h_{*} & =\left(i\left[t \cdot j m g_{1} \gamma\right], f^{\prime}\left[t \cdot j m g_{1} \gamma\right]\right) \sim\left(i t \cdot j i m g_{1} \gamma, f^{\prime} t \cdot j f^{\prime} m g_{1} \gamma\right) \\
& \sim\left(i t, f^{\prime} t \cdot j m^{\prime} f_{1} g_{1} \gamma\right) \sim\left(h_{1}, f^{\prime} t \cdot j m^{\prime} \gamma\right) \sim\left(h_{1}, h_{2}\right)=h .
\end{aligned}
$$

Suppose now that $k: X \rightarrow F$ is a map such that $\theta k$ is null-homotopic. Then $i k$ is null-homotopic so there is a continuous map $k^{\prime}: X \rightarrow \Omega(E, F)$ such that $m k^{\prime} \sim k$. Then

$$
m^{\prime} f_{1} k^{\prime}=f^{\prime} m k^{\prime} \sim f^{\prime} k \sim 0
$$

so there is a continuous map $k^{\prime \prime}: X \rightarrow \Omega E^{\prime}$ such that $i^{\prime} k^{\prime \prime} \sim f_{1} k^{\prime}$. Then $g_{2} k^{\prime \prime}$ maps $X$ into $\Omega E$ where $g_{2}: \Omega E^{\prime} \rightarrow \Omega E$ is the map induced by $g$. Hence

$$
0 \sim m^{\prime \prime g} k^{\prime \prime} \sim m g_{1} i^{\prime} k^{\prime \prime} \sim m g_{1} f_{1} k^{\prime} \sim m k^{\prime} \sim k
$$

and the indicated sequence is exact.

Consider the map $\sigma=f g \cdot j: E^{\prime} \rightarrow E^{\prime}$. Since $f$ and $g$ are fiber maps and $p^{\prime}$ is an $H$-homomorphism, then $p^{\prime} \sigma$ is null-homotopic. Hence there is a homotopy lifting $\sigma_{*}: E^{\prime} \rightarrow F^{\prime}$ such that $i^{\prime} \sigma_{*} \sim \sigma$. It follows that the map $\tau=\left(g, \sigma_{*}\right): E^{\prime} \rightarrow E \times F^{\prime}$ is a right homotopy inverse for $\varphi$. Although $\varphi$ may not be an $H$-homomorphism with respect to the product $H$-group structure for $E \times F^{\prime}$, the hypotheses of Theorem 2 are satisfied and Theorem 1 follows.

THEOREM 3. Let $(E, p, B)$ and $\left(E^{\prime}, p^{\prime}, B\right)$ be H-group fibrations over $B$ and $f: E \rightarrow E^{\prime}$ a base point preserving fiber H-homomorphism. If the restriction $f^{\prime}: F \rightarrow F^{\prime}$ is a homotopy equivalence and there is a base point preserving fiber map $g: E^{\prime} \rightarrow E$, then $f$ is a fiber homotopy equivalence.

Proof. Let $\tau=\left(g, \sigma_{*}\right)$ be the right homotopy inverse for $\varphi$ given in the preceding proof and let $h^{\prime}: F^{\prime} \rightarrow F$ be a homotopy inverse for $f^{\prime}$.

Define $h=j i h^{\prime} \sigma_{*} \cdot g: E^{\prime} \rightarrow E$. Then

$$
f h \sim j f i h^{\prime} \sigma_{*} \cdot f g=j i^{\prime} f^{\prime} h^{\prime} \sigma_{*} \cdot f g \sim j i^{\prime} \sigma_{*} \cdot f g \sim j \sigma \cdot f g \sim \mathrm{id}_{E^{\prime}}
$$


so that $h$ is a right homotopy inverse for $f$. To see that $h f$ is homotopic to $\mathrm{id}_{E}$, consider the map

$$
s=\left(h f \cdot j, c\left(e_{0}^{\prime}\right)\right): E \rightarrow E \times F^{\prime}
$$

where $c\left(e_{0}^{\prime}\right)$ is the constant map whose only value is $e_{0}^{\prime}$. Then $\varphi s$ is nullhomotopic so there is a continuous map $s_{*}: E \rightarrow F$ such that $\theta s_{*} \sim s$. Hence

$$
\theta s_{*}=\left(i s_{*}, f^{\prime} s_{*}\right) \sim s=\left(h f \cdot j, c\left(e_{0}^{\prime}\right)\right)
$$

Then $f^{\prime} s_{*}$ is null-homotopic and, since $f^{\prime}$ is a homotopy equivalence, it follows that $s_{*}$ is null-homotopic. Thus

$$
h f \cdot j \sim i s_{*} \sim 0
$$

so $h f$ is homotopic to the identity map on $E$. This completes the proof that $f$ is a homotopy equivalence. Since $f$ is a fiber map, the fact that it is a fiber homotopy equivalence follows from [2, Theorem 6.1].

Since the fiber map $g$ of Theorems 1 and 3 is not required to bear any particular relationship to $f$, one might guess that these results would still be true without the existence of $g$. The following example shows that this is not the case.

EXAmple. Consider the Hopf map $h: S^{3} \rightarrow S^{2}$ with basic fiber $S^{1}$. Since $\left(S^{3}, h, S^{2}\right)$ is a regular Hurewicz fibration, then $\left(\Omega^{2} S^{3}, \Omega^{2} h, \Omega^{2} S^{2}\right)$ is also a Hurewicz fibration. (Here $\Omega^{2}=\Omega \Omega$ is the iteration of the based loop space functor.)

Let

$$
\begin{aligned}
(E, p, B) & =\left(\Omega^{2} S^{3}, \Omega^{2} h, \Omega^{2} S^{2}\right) \\
\left(E^{\prime}, p^{\prime}, B\right) & =\left(\Omega^{2} S^{2} \times \Omega^{2} S^{1}, \text { projection, } \Omega^{2} S^{2}\right)
\end{aligned}
$$

and let $f: E \rightarrow E^{\prime}$ be the natural map induced by $h$. Observe that $F=F^{\prime}=$ $\Omega^{2} S^{1}$, a contractible space. Since $\Omega^{2} S^{3}$ and $\Omega^{2} S^{2}$ are not homotopy equivalent, then $E \times F^{\prime}$ and $E^{\prime} \times F$ are not homotopy equivalent. Observe also that the restriction $f^{\prime}: F \rightarrow F^{\prime}$ is a homotopy equivalence but $f: E \rightarrow E^{\prime}$ is not a homotopy equivalence.

THEOREM 4. Let $(E, p, B)$ and $\left(E^{\prime}, p^{\prime}, B\right)$ be H-group fibrations over $B$ and $f: E \rightarrow E^{\prime}$ a base point preserving fiber H-homomorphism. If the basic fibers $F$ and $F^{\prime}$ are contractible in $E$ and $E^{\prime}$ respectively and the induced map $f_{2}=\Omega f: \Omega E \rightarrow \Omega E^{\prime}$ is a homotopy equivalence, then the restriction $f^{\prime}: F \rightarrow F^{\prime}$ is a homotopy equivalence. 
Proof. Consider the diagram

$$
\begin{gathered}
\Omega E \stackrel{i}{\longrightarrow} \Omega(E, F) \stackrel{m}{\longrightarrow} F \stackrel{i}{\longrightarrow} E \\
l_{2} \uparrow \downarrow f_{2} \quad l_{1} \uparrow \downarrow f_{1} \quad \downarrow f^{\prime} \downarrow f \\
\Omega E^{\prime} \stackrel{i^{\prime}}{\longrightarrow} \Omega\left(E^{\prime}, F^{\prime}\right) \stackrel{m^{\prime}}{\longrightarrow} F^{\prime} \stackrel{i^{\prime}}{\longrightarrow} E^{\prime}
\end{gathered}
$$

where

$$
l_{1}: \Omega\left(E^{\prime}, F^{\prime}\right) \rightarrow \Omega(E, F), \quad l_{2}: \Omega E^{\prime} \rightarrow \Omega E
$$

are homotopy inverses for $f_{1}$ and $f_{2}$ respectively. (Recall from the proof of Theorem 1 that $f_{1}$ is a homotopy equivalence.)

Since the horizontal sequences are exact and since $F$ and $F^{\prime}$ are contractible in $E$ and $E^{\prime}$ respectively, there exist continuous functions $\chi: F \rightarrow$ $\Omega(E, F), \chi^{\prime}: F^{\prime} \rightarrow \Omega\left(E^{\prime}, F^{\prime}\right)$ such that

$$
m \chi \sim \mathrm{id}_{F}, \quad m^{\prime} \chi^{\prime} \sim \mathrm{id}_{F^{\prime}} .
$$

A straightforward argument involving the exactness and commutativity properties of diagram $\left({ }^{*}\right)$ shows that the functions

$$
f_{0}=m^{\prime} f_{1} \chi: F \rightarrow F^{\prime}, \quad g_{0}=m l_{1} \chi^{\prime}: F^{\prime} \rightarrow F
$$

are mutual homotopy inverses. Since $f^{\prime}$ is homotopic to $f_{0}$, then $f^{\prime}$ is a homotopy equivalence.

ReMARK. If $F$ and $F^{\prime}$ are contractible in $E$ and $E^{\prime}$ respectively, it follows from [1, Theorem 7] that we have homotopy equivalences

$$
F \times \Omega E \approx \Omega B \approx F^{\prime} \times \Omega E^{\prime} .
$$

With the algebraic properties of the spaces involved and the fact that the induced $\operatorname{map} f_{2}: \Omega E \rightarrow \Omega E^{\prime}$ is a homotopy equivalence, it is then natural to expect $F$ and $F^{\prime}$ to be homotopy equivalent.

The following result is an immediate consequence of Theorems 3 and 4:

COROLLARY. In addition to the hypotheses of the preceding theorem, suppose that there is a base point preserving fiber map $g: E \rightarrow E^{\prime}$. Then $f$ is a fiber homotopy equivalence.

COROLlaRY. In addition to the hypotheses of the preceding theorem, suppose that $B$ is arcwise connected and has a numerable covering $\left\{V_{\lambda}\right\}$ such that each inclusion $V_{\lambda} \rightarrow B$ is null-homotopic. Then $f$ is a fiber homotopy equioalence. 
Proof. By Theorem $4, f^{\prime}: F \rightarrow F^{\prime}$ is a homotopy equivalence. Since $B$ is arcwise connected, the desired conclusion follows from [2, Theorem 6.3].

\section{REFERENCES}

1. F. H. Croom, Exact loop space sequences, Fund. Math. (to appear).

2. A. Dold, Partitions of unity in the theory of fibrations, Ann. of Math. (2) 78 (1963), 223-255. MR 27 \#5267.

Department of Mathematics, University of Kentucky, Lexington, Kentucky 40506

Current address: Department of Mathematics, University of the South, Sewanee, Tennessee 37375 